Relations industrielles

Industrial Relations

\title{
Concentration in the Manufacturing Industries of the United States, Ralph L. Nelson, A Midcentury Report - New Haven and London, Yale University Press, 1963, 288 pages.
}

\section{Fernand Blais}

Volume 20, numéro 4, 1965

URI : https://id.erudit.org/iderudit/027627ar

DOI : https://doi.org/10.7202/027627ar

Aller au sommaire du numéro

Éditeur(s)

Département des relations industrielles de l'Université Laval

ISSN

0034-379X (imprimé)

1703-8138 (numérique)

Découvrir la revue

Citer ce compte rendu

Blais, F. (1965). Compte rendu de [Concentration in the Manufacturing Industries of the United States, Ralph L. Nelson, A Midcentury Report - New Haven and London, Yale University Press, 1963, 288 pages.] Relations industrielles / Industrial Relations, 20(4), 717-718.

https://doi.org/10.7202/027627ar

Tous droits réservés @ C Département des relations industrielles de l'Université Laval, 1965
Ce document est protégé par la loi sur le droit d'auteur. L'utilisation des services d’Érudit (y compris la reproduction) est assujettie à sa politique d'utilisation que vous pouvez consulter en ligne.

https://apropos.erudit.org/fr/usagers/politique-dutilisation/ 
Enfin, la section trois aborde le processus de balancement.

Cette monographie apporte donc une contribution importante à ce champ de recherche et donne un compte-rendu des explorations et résultots obtenus jusqu'ici.

\section{Fernand Blais}

Emplois et gains dans les professions scientifiques et techniques, 1959-1962, Les travailleurs intellectuels: rapport no 14, Hon. Allan MacEachen, George V, Haythorne, Direction de I'Economique et des Recherches, Ministère du Travail, Ottawa, octobre 1964, 26 pages.

Ce rapport du Ministère fédéral du travail donne les gains et l'emploi dans les professions scientifiques et techniques, de 1959 à 1962 , et vient compléter une série de données publiées antérieurement dans le rapport no 12, de la même série, sur les travailleurs intellectuels (ovant-propos).

Recueillies par échontillonnage, des données sur l'instruction, l'emploi et les gains sont disponibles depuis 1958. Elles fournissent des renseignements sur le marché du travail dans six domaines de spécialisations: l'agriculture, l'architecture, le génie, les sciences forestières, les sciences naturelles et la médecine vétérinaire. Le rapport est centré davantage, cependant, sur le génie et les sciences seulement, mais les renseignements conservent six variables: l'âge, l'instruction, le nombre d'années depuis le boccolouréot, le genre d'employeur, la région d'emploi et la fonction. (p. 5)

\section{Fernand Blais}

\section{Poor Countries and Authoritarian Rule,} Maurice F. Neufeld, New York State School of Industrial and Labor Relations, Cornell University, Ithaca, New York, 1965, 240 pages.

Les pays pauvres connoissent des problèmes économiques difficiles. Ils possèdent de faibles taux de croissance et le fossé avec les poys riches s'accentue toujours. L'ignorance de ces peuples demande aussi des transformations radicales.

Lo situation est urgente et les leaders politiques qui ont pris le pouvoir ovec la promesse d'une perspective économique meilleure souffrent, en courte période, des reproches de lo population.
Pour foire face à ces reproches, ces derniers ont tendance à délaisser la forme de gouvernement démocratique pour s'orienter vers un grouvernement autoritaire, en développant un système politique ò parti unique et en exerçant une coercition gouvernementole très poussée.

II va sans dire que ces changements vont influencer toute la vie socio-économique de ces pays de même que les relations internationales pour le reste du siècle.

Ainsi, Neufel a développé neuf propositions qui tentent d'expliquer le cheminement de ces pays vers l'état autoritaire sinon dictatorial. Il a commencé à. développer ces propositions lors de son étude: \& Italy: School for Awokening Countries », bien qu'ò ce moment là elles étaient peu clarifiées. Aujourd'hui, il développe ces propositions dans des chapitres différents, traitant ainsi de la liberté, du nationalisme, du syndicalisme, des gouvernements et des relations extérieures.

Certains diront que I'histoire de ces pays conquis d̀ l'indépendance est relativement jeune. Mais elle est déià assez longue pour expliquer le développement de certaines formes autocratiques dans les gouvernements. D'ailleurs l'auteur explique ce développement, par ses neuf propositions, comme un processus continu et quasi inévitable, à moins d'un renversement inattendu dans le domaine économique. Toutefois, ces propositions s'appliquent à des degrés différents selon les pays et selon des circonstances, tout en étant un reflet assez fidèle de la situation de ces pays.

\section{Fernand Blais}

\section{Concentration in the Manufacturing Indus-} tries of the United States, Ralph L. Nelson, A Midcentury Report - New Haven and London, Yale University Press, 1963, 288 pages.

$1 /$ peut paraître inévitable que le contrôle d'une industrie soit dans les mains de quelques-uns à un moment ou l'autre de son évolution. La monographie, por Nelson, sur la concentration dans l'industrie manufacturière aux Etats-Unis, révèle justement que cette concentration varie dans le temps.

Cette étude faite à partir de données non-publiées du Bureau de la Statistique, tente de conserver le problème des monopoles dans un contexte adéquat, de même qu'elle fournit des renseignements sur le processus de concentration et son influence sur le modèle de croissance de cette industrie. 
RELATTONS Industrielles, vor. 20 , No 4

De plus, on peut observer l'effet des classifications sur les modeles de concentration. La définition que l'on donne à chaque industrie influence lo mesure de concentration de telle sorte qu'il est nécessaire de raffiner la technique de classification pour empêcher les biais systématiques.

Enfin, l'étude permet de caractériser les leaders de l'industrie, quant d̀ leur importance et leur degré de concentration et examiner si ces différences sont cousées par le. degré de concentration.

Ainsi, ce volume tente d'extentionner la variété des mesures de concentration à l'étude de la structure de l'industrie plutôt que de les limiter d la simple étude du degré de compétition dans une industrie.

Puisque les politiques gouvernementales dépendent souvent de la compréhension d'un tel phénomène, l'analyse de R. Nelson a donc des implications pratiques pour la formulation de ces politiques.

\section{Fernand Blais}

Les organigrammes, $2 e$ édition, Entreprise Moderne d'Edition, Paris, 1964, 131 pages.

Dans toute entreprise l'organigramme se révèle un outil précieux. " Il oblige à une définition précise des postes et des fonctions et permet une meilleure coordination des différents services\$. II reflète donc lo structure de l'entreprise et doit être constomment mis à jour à mesure que celle-ci prospère

$\mathrm{Ce}$ monuel est alors un document renseignant sur les différents types d'organigrammes, que ce soit pour les secteurs privé ou public ou encore pour les secteurs primaire, secondaire et tertiaire.

Ainsi dans la première partie, l'outeur définit l'organigramme, son utilité, son étoblissement; et propose une méthode quel que soit le moment de sa rédaction. Dans la seconde, nous retrouvons les types d'organigrommes fronçois et étrongers, tous troduisant une réalité. Certes on ne peut les oppliquer intégralement d'une entreprise dे l'autre, car il n'y a pas d'organigramme posse-portout.

Comme conclusion, l'outeur rappelle * qu'une organisotion s'adapte d une chose qui est en perpétuelle évolution cor cette chose produit et lo production est très fluente, puisqu'elle répond à des besoins en perpétuelle contingence. Donc l'organigram- me n'est pos un instrument établi a ne vorietus $\otimes$.

Fernand Blais

L'entreprise et la culture générale, $P$ Demarne, L. Meyer, J. Quinson, M. Raclot L. Salleron, Entreprise Moderne d'Edition, 4, rue Combon, Paris 1, 1965, 147 pages

* Le problème de la culture générole est-il de ceux dont les entreprises aient à se soucier? Est-ce de son devoir et dans soir intérêt?

Cet ouvrage tente de répondre à ces questions et présente cina études sur des aspects différents.

La première partie, par Louis Salleron, veut définir \& culture générale» et fournit des suggestions pour une action meilleure Selon l'auteur, le chef d'entreprise est parfoitement en mesure de fovoriser chez ses collaborateurs le développement de la culture générale.

Louis Meyer et Pierre Demarne, deux individus engogés l'un pour la C.S.F., l'autre pour IBM-France, discutent, au cours d'une rencontre, du problème de la culture chez les codres et relatent différentes expériences qu'ils ont connues.

Michel Radot présente l'expérience de la société Lorroine-Escout dans le domaine culturel

Enfin Jean Quinson expose le progromme de formation générale de la S.N.C.F.

Ce volume propose donc des thèmes de réflexion sur le sujet et donne certaines expériences françaises et étrangères dans le domoine. L'intense majorité des travailleurs désire se cultiver et si l'entreprise n'y trouve pas un intérêt immédiat, l'économie générale bénificie toujours d'un niveau d'éducation plus élevé. . Ce sont des raisons suffisantes pour prendre conscience du problème et essayer de faire quelque chose $s$. (p. 58)

\section{Fernand Blais}

\section{Problems of Employment in Economic Deve-} lopment, International Institute for Lobour Studies, Reprinted from International Labour Review, Vol. LXXXIX, No. 3, mors 1964, Genève, 75 pages.

L'International Institute for Labour Studies présente quatre articles sur les problèmes 